\title{
The Epistemology of the Very Small
}

\author{
Joseph C. PITT \\ Department of Philosophy, Virginia Tech \\ jcpitt@vt.edu
}

\begin{abstract}
The question is how do Scanning Electron Microscopes (SEMs) give us access to the nano world? The images these instruments produce, I argue, do not allow us to see atoms in the same way that we see trees. To the extent that SEMs and STMs allow us to see the occupants of the nano world it is by way of metaphorical extension of the concept of "seeing". The more general claim is that changes in scientific instrumentation effect changes in the concepts central to our understanding of scientific results.
\end{abstract}

\section{Introduction}

The world of nanotechnology is the world of the very small. According to Eugene Wong in his testimony to the Subcommittee on Basic Research of the U.S. House of Representatives Committee on Science in June 1999,

One nanometer is 1-billionth of a meter. To get an idea of the size, we can compare some familiar things. The diameter of an atom is about $1 / 4$ of 1 nanometer. The diameter of a human hair of 10,000 nanometers. The protein molecules, which are so important, so critical to life, are several nanometers in size. Moving to man-made things. The smallest devices on commercially available chips are about 200 nanometers, whereas the smallest experimental chips are approximately 10 nanometers in their smallest dimension. (Nanotechnology, p. 3)

The question I want to investigate here is "how can we come to know what is going on in this domain of tiny things?" There are a couple of issues to be examined: (a) what do we mean by "know"? and (b) how do we access this domain? Some would argue that the two are separate - that we can come to an agreement on the meaning of "knowledge" independently of settling the question of how we can access the nano-world. I want to argue that this is not the case. What we come to know about the nano-world is very much a direct function of how we access it and the criteria we bring with us that allow us to evaluate that access. This claim is part of a larger thesis: that we also modify our conception of knowledge as we develop criteria for calibrating our instruments.

\section{Seeing the Unobservable}

One would think that there really isn't a problem here since, for the last 60-70 years in the philosophy of science there has been an on-going argument over the status of objects smaller than what we can see with the naked eye. Basically the question to be answered is this: if you can't see it, is it real? The question actually is somewhat more complicated than the formulation just provided. It is usually couched in the context of determining whether or not entities proposed by true scientific theories exist. This question cannot be reduced to the question of observability alone, for not all theoretical entities are unobservable, e.g., galax- 
ies, and not all unobservable theoretical entities are very small, e.g., black holes. Further, scientific theories are not the sorts of things of which we know with absolute confidence that they are true. They are constantly being challenged, modified, changed, and revised. Further, given the constant state of flux of theories in use, no one really knows what the theory is at the time at which it is being worked out. We finalize the content and form of a theory only after we have rejected it and moved on to something else - we finalize these versions of theories in textbooks. ${ }^{1}$ All this being the case, it is no wonder that the status of theoretical entities, entities proposed by scientific theories that have yet to be proven to be completely true, is in question, in particular, those very small entities, the ones we can't see.

But it might be objected, that we can see them by way of various microscopes - devices that by their very names are designed to scope (see) the very small (micro). Here is where things get sticky, however. The crux of the matter has to do with the meaning of "to see". The meaning of the verb "to see" has changed over time. Further, I would argue, what it means to see something has changed precisely because we have developed instruments to help us "see" more and more in different ways. Moreover, we have come to call this "seeing" without attending to the fact that it is not "seeing" in the usual way. Further, because we are usually inattentive to that fact, we fail to capture the nuances of the conceptual difficulties we should encounter when we talk about seeing things through a microscope. Let me explain.

\section{The Role of Metaphor}

The sense in which we "see" though a microscope is different than the sense in which we "see" a tree or a coffee cup. Or to put it another way, we have extended the meaning of the verb "to see" to accommodate our use of microscopes. Or to put it a third way, to talk about "seeing through a microscope" is to employ a metaphor. A metaphor is a way of easing our way into an understanding of the unknown by applying the familiar to the unfamiliar. We call a number of things "seeing" today because we metaphorically equate what we are doing with seeing as we naturally understand it.

For example, seeing through a microscope differs from seeing a tree with the naked eye because we don't have to learn how to see a tree. We may have to learn that that thing there is a tree, which is learning how to use our language - but you can run into a tree and hurt yourself and know that that thing there is what hurt you and not know that it is a tree. But can you do that when looking through a microscope? I would argue "no". It is not because you cannot run into microscopic entities - it is rather that you can't see them at all until a couple of things happen that aren't required for seeing in the macro-world (that is, the world of tables, chairs, trees - the world in which we live): (i) you have to learn how to use the instrument; (ii) you have to learn how to see what is there.

\section{Learning to See through Microscopes}

Learning how to see through the microscope for the first time is difficult. You have to learn how to do a number of things, for example, not to get your eye too close to the lens, and keeping your head still and turning the focus knob at the same time. Those things take a little while to master. But the truly hard part is learning to see what is on the slide. This problem was with us from the start. Consider what Hooke had to say in the Micrographia in 1665 .

What each of the delineated Subjects are, the following descriptions annext to each will inform. Of which I shall here, only once for all add. That in divers of them the 
Gravers have pretty well followed my directions and draughts; and that in making of them I have endeavored (as far as I was able) first to discover the true appearance, and next to make a plain representation of it. This I mention the rather, because of these kinds of Objects there is much more difficulty to discover the true shape, then of those visible to the naked eye, the same Object seeming quite differing, in one position of Light, from what it really is and may be discover'd in another. And therefore, I never began to make any draught before by many examinations in several lights, and in several positions to those lights, I had discover' $d$ the true form. For it is exceeding difficult in some Objects, to distinguish between a prominency and a depression, between a shadow and a black stain, or a reflection and a whiteness in the colour. Besides, the transparency of most Objects renders them yet much more difficult then if they were opacous.

Leeuwenhoek who is sometimes called the father of the microscope, complained of the same problem in a letter to Oldenburg: "...some of the forms I see are so fine and small, that I don't know how even a good draughtsman could trace them, unless he make them bigger."

But yet we have learned how to see using a microscope - partially it required the development of cell-theory and later, the theory of crystals. That is, once we had a way of understanding the sorts of things we were looking at, we had the means to see them as separate and distinct items, possessed of various properties, shapes, and appendages. This requires theory. It is not enough to know how to use a microscope, one must know what to look for. What to look for is dictated by various theories about the domain of the small.

But even the possession of theory is not enough, we also must develop the means of separating out individuals one from another. In the case of biological organisms, for example, we rely on staining techniques. And further, we had to learn to rely on the credibility of staining techniques. This is not a trivial matter. Let me relay a true story. Mike was a MS student in biology working on the eye of the Hackfish. He was having trouble staining his slides, so when he had the opportunity to attend a conference where he could ask for some help he leapt at it. At the conference he managed to corner the acknowledged expert on staining slides and explained his problem. The expert reportedly told Mike the secret to success: "first, turn off all the lights in the lab and make sure the windows are darkened. Then close your eyes and raise your left foot. Then, hopping on your right foot, make a 360 turn to the left. Then lift your right foot and do a 360 to the right. Then stain your slides." Mike was crushed. After he returned from the conference we had numerous discussions about what kind of a message the great man could have thought he was conveying, but never figured it out. Mike finished his degree, but he had lost his faith in science and left to go work for British Petroleum.

The moral of the story I take to be this: some of what we do in the process of seeing the very small involves a skill that cannot be taught by rote. That being the case, you would expect the results of using stains on slides to be doubtful, but, interestingly, they are not. Part of what is involved in seeing with a microscope involves accepting the fact that some people are better at staining slides than others, and we rely on them to prepare the slides. In a crucial way we have extended the concept of seeing by accepting the fact that it may take more than one person for seeing to occur and, further, that not both might actually do the seeing.

In addition to learning to rely on staining techniques to provide us with access to the very small, we also have to accommodate what I will call the problem of focus. Prior to 1702, focusing was done the old fashioned way: you brought the object to be examined into focus by holding the object in one hand, the lens through which you were looking in the other, and adjusted them until something recognizable came into view. According to Gerard L'E. Turner, Leeuwenhoek's microscope was “A tiny lens contained in a metal plat, with a 
spike to hold the specimen close to the lens; the instrument was then handheld immediately in front of the eye" (in Bud and Warner 1998). James Wilson, an Englishman, developed the screw-barrel roughly forty years later in 1702. The screw-barrel allowed for mechanical focusing. With the development of mechanical focusing, stability became a factor that could be mastered. So, as we have seen, learning how to see through the microscope involved a number of steps, advances in theory, skill, and in the mechanical arts themselves.

I would like to look closer at the problem of focusing. Learning to focus an instrument is now an accepted part of seeing. But consider how strange this is. You don't have to be taught to focus your eyes to see macro objects like tables and mountains. What occurs is a natural phenomenon. Our biology takes over. And when you think of it, it is a rather amazing feature of our bodies. Focusing a seeing instrument, however, is an unnatural act. And yet, because it is integral to seeing with that instrument, it has become accepted as part of what we do when we use an instrument to see. And it is all part of the extended metaphor we now employ when we talk about seeing through a microscope or a telescope. It includes staining slides (or in the case of a telescope, computer enhancing photographs or using color filters), focusing instruments, theory, etc., all by way of accommodating what we do to what our eyes do.

\section{Learning to See with Electron Microscopes}

In his fascinating study Picture Control, Nicolas Rasmussen examines in great detail a number of these issues as they pertain to the electron microscope. In particular, he focuses on how criteria for acceptance are established, that is, on the social domain. Allow me to offer a lengthy quote:

[...] early biological electron microscopy involved a struggle for picture control on a number of levels. ...picture control figured in a biologist's subjective experience of the electron microscope as one of three relevant readouts, and along with focus, one of the two open to intervention. Of course, there was no such thing among the seven indicators and nineteen switches and knobs on the console of the Radio Corporation of America (RCA) EMU microscope [...] Control of who could make pictures with the electron microscope, how pictures should be made, what pictures would be printed, and how those pictures ought to be used in establishing biological facts were the dominant issues when the new instrument was introduced to biologists at the onset of the Second World War [...] By the end of the war, a community of scientists in whom expertise was vested [...] was established, and assumed a basic level of regulatory control. But for individual microscopists, control of the characteristics and interpretation of pictures remained a problem, and one that was divergently addressed in different biological subfields, even in different research programs within them. (Rasmussen 1997, p. 1)

Now Rasmussen is talking about the social evolution of standards in the same breath as the social evolution of consensus over who had access to the machines etc, and it sounds very social constructivist. The battles and issues he identifies are appropriately discussed as issues of power, access, and interpretation. Perhaps key among them is power. For what we are talking about is who sets the criteria and on what grounds. But no matter what the politics may be, there is a world out there that sets the bottom line. Or does it?

It is at this point that we need to distinguish between optical and electron microscopy. With optical microscopes we are actually looking at something. We prepare a slide by putting something on it. Further we are aware of the fact that when, for example, we stain a slide, we have introduced something to the slide and we can test to determine how that affects the specimen. What exactly we are seeing is a function of how we interpret what we 
see using theory, but that there is something there to see is clear. With an electron microscope, on the other hand, we do not "see" the specimen. The machine uses an extremely fine point on a stylus to reveal the contours of a surface without actually touching the surface. Instead of dealing with the physics of light and the properties of specimens as we do with an optical microscope, with the electron microscope we get a "picture" of that surface through the use of various computer programs which take the input from the stylus running over the surface, then use the physical theory of the properties of matter to "interpret" the results, thus producing an image.

The question here is the extent to which the machine creates the phenomena. There is a weak and a strong version of this claim. The weak version holds that without the machine we would not be able to see what we see. This would suggest that the things we see with the machine are there in the world, but we don't have the means to access them without the machine. That claim is fairly innocuous. The problem arises because of the stronger interpretation of the claim that the machine creates the phenomena, which is: what we see is an artifact of the machine itself - if doesn't exist in the real world until we have the machine. If that is true, then the next question becomes "well, what kind of a thing is it? Does it exist or not?" To address this let us consider in slightly greater detail what it is that an electron microscope does.

Rasmussen and Hawkes give a rather succinct account that will assist us:

An electron microscope produces a magnified image through a specimen's interaction with a beam of high energy electrons, usually 50-200 kilovolts. There are two principle forms of this instrument. In a transmission electron microscope (TEM), an electron beam at least as large as the imaged area passes through the specimen and forms an image on a fluorescent screen or photographic film. In a scanning electron microscope (SEM), an electron beam that is small compared with the imaged area passes over the specimen in a regular pattern, and a picture of the specimen surface is reconstructed on a video tube. Image contrast is formed in many ways. In the TEM, electrons are deflected by atoms inside the specimen, without absorption, creating a shadow pattern of greater and lesser electron transmission. In the SEM, interaction of the beam with the specimen surface produces varying intensities of backscattered and secondarily released electrons for each position in the scan, and these are registered by a detector placed appropriately near the specimen. (in Bud \& Warner 1998, p. 382)

In each type of electron microscope, we end up with an image. But it is not an image directly obtained by seeing. The image is the result of a process in which the object under examination is not "caught" but rather reflected. But it is not reflected as a mirror reflects your face. It is a secondary reflection, almost like trying to draw the right hand wall of a handball court by observing where the ball lands on the front court after angling it off the right hand wall. The assumption is that the image represents the object. But it is not a representation such as we find when we draw a picture or produce a painting, say, a still life. And yet, we are content to say that the images are reasonable pictures of the objects - even though we can't see the objects directly. Under normal circumstances, common sense would contest the claim that an image produced by an electron microscope is an accurate representation of a very small object that cannot be seen. But we accept the claim. Why? The question becomes more demanding when we consider some further complications. Rasmussen and Hawkes lay out some of the problems for seeing biological specimens:

The electron beam demands a vacuum, so specimens cannot be alive and require drying in some minimally destructive way. Since electrons interact strongly with matter, the beam penetrates only very thin specimens. Moreover, the beam heats specimens, and so can alter volatile biological materials. Contrast is another obstacle, since the 
different substances in living things vary little in opacity to electrons. (in Bud and Warner 1998, p. 384, emphasis added)

So an early major problem was the modification of the specimen by the electron beam. The solution was to find a way to fix the specimen. In the biological sciences the solution was initially chemical, then supplemented by freezing. In the physical sciences this involved the development of techniques for coating the specimen with a thin film.

What is of interest to us is the fact that the development of means to stabilize the specimen did not alter the initial problem of the manner in which the electron microscope produces an image. The reliability of the image was not the issue, the stability of the specimen was. Essentially, we find the same situation as with the optical microscope: an evolving set of techniques and standards that fundamentally change our conception of seeing. But, what is interesting is that the sense of seeing evolves together with the standards and techniques. This results in a consensus on what a good image looks like, even though it is not an image in the earlier, pre-electron microscope, sense.

\section{The Nano Scale and Nano Technology}

So let us now return to the nano scale and nano technology. Nano technology is the construction of very small artifacts and systems of artifacts. It is miniaturization taken to the max. ${ }^{2}$ And our question is how do we know that the things are working at the nano level as they are supposed to? One way is to look and see. And this is what we cannot do with electron microscopes or STMs without begging the question. A second way, much more economical and intellectually sound, is to wait and see whether what these mini machines are supposed to do actually happens. It is a pragmatic solution. William James' most notable contribution to philosophy was the aphorism: For a thing to make a difference, there must be a difference. I do not believe that we will have a problem knowing whether the nano machines are doing their job.

However, our understanding of our interaction with the nano world shares similar characteristics with what we mean when we see through a microscope. I quote again from the Congressional hearings on nanotechnology, and ask you to listen to the language carefully. Richard Smalley, Nobel Laureate, is discussing the impact of carbon nanotubes. He is discussing a slide he has put up on the screen.

As individual nanoscale molecules, these carbon nanotubes are unique. Just think of one at a time. They have been shown - here you see one draped across a few electrodes. They have been shown to be true molecular wires, to conduct electricity like copper - in fact, even better - and have already been assembled into the first molecular transistor ever built; with just a single molecule. (Nanotechnology, p. 9)

What struck me was the casual manner in which Smalley refers to seeing a single molecule. The idea that a single molecule could be a transistor is itself difficult to grasp. More significantly, the ease with which he speaks of seeing the molecule is of a piece with how he speaks of manipulating them. It is both natural and, in the context of what we mean by "see", illustrative of the point I have been trying to make. The methods, standards and implications of modifying the language to accommodate the new technology comes slowly but of a piece.

The stronger thesis that it is a metaphorical extension of standard usage will have to wait for another time for its defense. But just consider another familiar nanotechnology claim. This simple statement, so straight forward, and yet so misleading, makes the point. I know what it means to divert a small stream of water threatening to destroy my driveway by removing a tree limb that has blocked a drainage ditch. I pick it up and toss it into the 
field. By analogy I think I know what it means to put an atom where you want it to go, but I doubt that it is as simple as picking up a stick. Yet, the language of "putting atoms where you want them to go" makes it sound so familiar. What is really entailed? All we are talking about is manipulating atoms. Atoms, remember, are $1 / 4$ of a nanometer in diameter. A nanometer is 1 billionth of a meter. To unpack the claim about putting atoms where you want them means understanding a lot about the means we have devised for doing this sort of work, the tools we have built and the assumptions we employ about what we are doing. My guess is that putting molecules where you want them is much like seeing through a microscope, it is now a team activity, involving sophisticated instruments and subsidiary techniques, a lot of theory, many theories, a lot of skill, and a lot of luck.

That seeing in the context of using SEMs and very large telescopes has become a team activity is not in itself something negative. The point here is that it is a different sort of thing than seeing a tree. It is important to note this difference because it helps us understand how science changes. In particular, what has changed is not just that what we mean by "see". The introduction of these instruments also changes how we do science. This is not the obvious point that science is increasingly a team activity, it is that we have a new way of understanding scientific change. The moral of the story is that the older theories of scientific change proposed by Kuhn, Lakatos, and Laudan, seen in the light of the impact of new and innovative technologies such as scientific instruments, are deeply flawed. Scientific change is not merely a matter of the logical conditions under which scientific theories can be abandoned or accepted. It is a far more complicated process heavily influenced by the role of innovative instruments and other technologies that not only change the nature of the enterprise, but change the meaning of concepts like scientific observation, evidence, experiment. ${ }^{3}$ The impact of the new techniques required for a robust set of nanotechnologies will be important to watch as they will make a difference also in the manner in which we do the science of the very small.

\section{Notes}

1 It is an interesting feature of undergraduate science education that undergraduate students are rarely, if ever, taught the latest, most up-to-date theories. The textbooks, I would argue, are out of date by the time they are published. This is one reason why getting undergraduate science students involved in research in an active laboratory is so important to the future of the scientific enterprise.

2 It is important to note that this is as far as we can go in miniaturization given our current state of technology since the next level down is the quantum level, where stability of the material is itself in doubt.

3

For an elaboration of this theme see my Thinking About Technology.

\section{References}

Bud, R. \& Warner, D. (eds.): 1998, Instruments of Science, New York: Garland Publishing.

Nanotechnology: 1999, The State of Nano-science and its Prospects for the Next Decade. Washington: U.S. Government Printing Office.

Pitt, J.C.: 2000, Thinking About Technology. New York: Seven Bridges Press.

Rasmussen, N.: 1997, Picture Control: The Electron Microscope and the Transformation of Biology in America, 1940-1960, Stanford: Stanford University Press. 